\title{
Development of a low-cost confocal instrument to measure the axial dimensions of components in the anterior section of the eye
}

This article was published in the following Dove Press journal:

Clinical Optometry

29 July 2010

Number of times this article has been viewed

\author{
Kim K Buttenschön' \\ John M Girkin' \\ Daniel J Daly² \\ 'Centre for Advanced \\ Instrumentation, Durham University, \\ Durham, UK; ${ }^{2}$ Lein Applied \\ Diagnostics, Reading Enterprise \\ Centre, Reading, UK
}

\begin{abstract}
We report on the development of a non-contact, low cost instrument based upon confocal microscopy that is capable of rapidly and accurately measuring changes in the axial dimensions of components in the anterior section of the eye. The performance of the instrument was tested by applying an oil film to a vertically mounted artificial cornea to simulate a breaking up tear film. By measuring the influence of lubricating eye drops on the thickness of the human cornea and tear film in vivo with a commercial, interferometer based instrument, we show the need for higher resolution instruments. It was found that the new instrument measured a change in thickness as small as $5 \mu \mathrm{m}$ in the artificial system with a standard deviation of less than $1 \mu \mathrm{m}$. The change of thickness in the in vivo cornea was measured accurately with the interferometer based instrument, but no distinction between the thickness of the cornea and that of the tear film was possible. We therefore believe that the new confocal instrument and its further developments will play an important role in the fast and accurate measurement of tear film and corneal thickness and also in relation to glaucoma screening with applanation tonometry.
\end{abstract}

Keywords: corneal thickness, tear film, confocal microscopy

\section{Introduction}

The tear film is a crucial part of the human eye. It not only acts as a lubricating layer and provides nutrients for the cornea, ${ }^{1}$ but it also plays an important role in keeping the eye surface clean and preventing infections. ${ }^{2}$ As importantly, it is the first refractive surface, thus providing a main part of the refractive power of the eye. ${ }^{3}$ A healthy tear film also creates a smooth layer covering the rough, irregular surface of the corneal epithelium. ${ }^{4}$ This reduces scattering from the cornea, improves contrast in the detected image, and thus improves vision significantly. ${ }^{5}$

If there is any disturbance to the system such as the tears evaporating too rapidly or insufficient tears being produced, the tear film becomes ineffective and dry eye syndrome (DES) can develop. ${ }^{6}$ This not only leads to swollen, red and tired eyes, affecting the quality of life of the patient when performing daily tasks such as reading, using a computer or driving, ${ }^{7}$ but in more severe cases it can also lead to ulcers and scarring of the cornea and thus to some loss of vision. ${ }^{8}$ Fortunately, minor forms of DES can be easily treated by applying lubricating eye drops.

Due to conditions such as dry eye syndrome and the desire to study the anterior aspects of the eye in relation to laser-based refractive surgery as well as for glaucoma screening in relation to intraocular pressure measurements, accurate and reliable non-contact measurements of distances within the anterior chamber of the eye are playing a growing role in the diagnosis and monitoring of disease states within oph-
Correspondence: Kim K Buttenschön Department of Physics, Durham University, South Road, Durham DHI 3LE, UK

Tel +44 I9I 3343532

Fax +44 I9| 3343609

Email k.k.buttenschon@durham.ac.uk 
thalmology. Optometrists in the UK are now advised to take a corneal thickness measurement when performing glaucoma screening. ${ }^{9}$

We have developed an instrument capable of accurately measuring the axial dimensions of the anterior components of the eye through to the rear of the lens with a particular emphasis on the corneal and tear film thickness. In order to demonstrate the resolution and speed of the instrument we illustrate the performance by showing the thinning of an oil film on an artificial cornea. To demonstrate the need for further developed instruments in this area we present measurements taken with the LenStar (Haag-Streit AG, Koenitz, Switzerland) showing the influence of optive ${ }^{\mathrm{TM}}$ lubricating eye drops (Allergan, Inc, Irvine, CA, USA) for treating dry eye syndrome on the cornea and tear film thickness.

\section{Experimental set up}

The optical configuration of the instrument is shown in Figure 1. It is based upon a confocal microscope but rather than recording a series of optical sections to construct a three dimensional image, the instrument is configured to record a depth profile ${ }^{10}$ through the anterior section of the eye. The instrument thus provides the depth information required to measure features in the eye at high speed and with minimal data storage. Light from a low power laser diode (Laser 2000, Ringstead, Northamptonshire, UK) operating at $670 \mathrm{~nm}$ is initially collimated and circularized using integrated microoptics. After leaving the laser casing, the collimated light passes through a condensing lens, which creates a reference focal spot. It then expands through a polarizing beam splitting cube and a second beam splitting cube, which diverts a small proportion of the return beam onto a web cam for the monitoring of eye alignment. The expanding beam then strikes a lens combination, which collimates the light to a $12 \mathrm{~mm}$ diameter beam. The re-collimated beam passes through a quarter wave plate, rotating the polarization of the beam, before being focused onto the eye using a final lens. This final lens is mounted on a motorized translation stage (SMAC Europe Ltd, Crawley, West Sussex, UK), which moves the lens along the optical axis of the eye, giving a scanning depth of $5 \mathrm{~mm}$. A fixation target is introduced using a further beam splitter at the front of the instrument to help in minimizing eye movement.

Around $10 \mu \mathrm{W}$ of optical power is incident on the cornea and the reflected light is re-collimated before passing back through the quarter wave plate and subsequently being focused onto a silicon photo-diode mounted behind a confocal pinhole. The double pass through the quarter wave plate, in combination with the polarizing beam-splitting cube before the detector pinhole, improves the signal-to-noise ratio of the instrument helping to remove unwanted reflections reaching the detector. The slight polarizing properties of the cornea

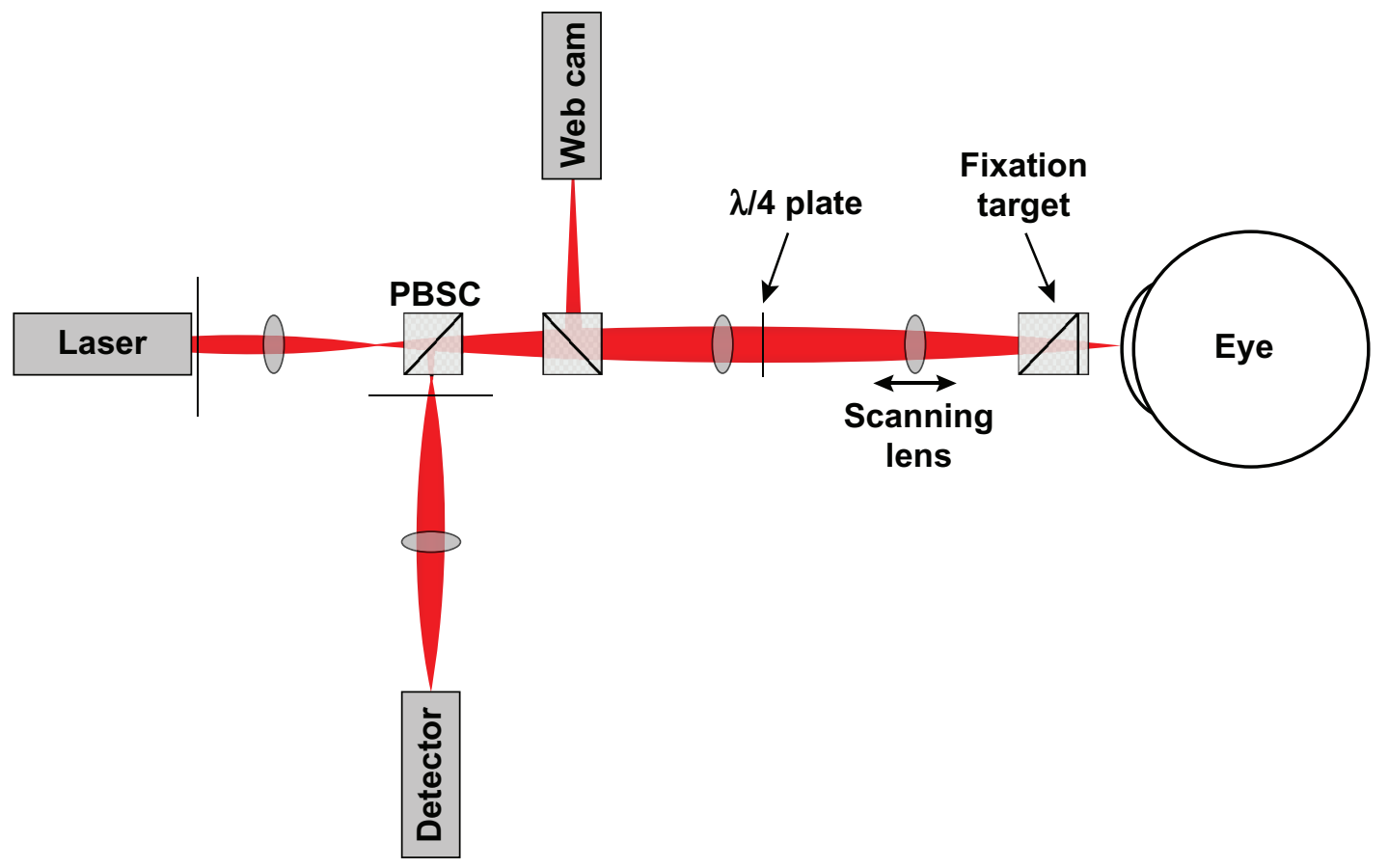

Figure I Schematic diagram showing the confocal instrument. The laser is focused down to a reference focal spot before expanding through a polarized beam-splitting cube (PBSC) and a second beam splitting cube which diverts a small part of the return beam onto a web cam. The light is then collimated and passes through a quarter wave plate before being focused onto the eye using a scanning lens. A further beam splitting cube is introduced at the front of the instrument to overlay a fixation target onto the beam. 
were not noted to have any effect on the performance of the instrument.

The SMAC stage holding the scanning lens has a resolution of $0.5 \mu \mathrm{m}$ and scans with a speed of $50 \mathrm{mms}^{-1}$. The scanning lens can be exchanged to alter the axial resolution of the system. The lens currently incorporated in the system has a focal length of $45 \mathrm{~mm}$ and a numerical aperture of 0.14 giving an axial resolution of approximately $64 \mu \mathrm{m}$. The working distance of the instrument is $30 \mathrm{~mm}$ from the last optical surface to the eye.

The data from the photo-diode was acquired using a National Instruments A/D card (DAQCard-6036E) and integrated into the control of the system using a custom written LabVIEW program, which also controlled the SMAC stage. One measurement was produced by averaging over 30 consecutive individual scans, with data being taken in both directions of the scan.

Figure 2, illustrating a typical scan of the cornea with the new instrument, shows the reflection from the air to cornea (air to tear film) interface and the smaller reflection from the posterior cornea. The experimental measurements were taken using the new instrument and the LenStar, which is an interferometer based instrument.

\section{Results}

To test the performance of the instrument, an oil film (immersion oil, $\mathrm{n}=1.51$ ) of unknown thickness was applied to an artificial cornea (PMMA, $\mathrm{n}=1.49$ ) to simulate an environment with rapidly changing dimensions (eg, the tear film). The cornea was manufactured such that the curvatures match the human cornea as closely as possible. It was mounted vertically so the oil could flow off. Figure 3 shows the typical trend of the influence of the oil on the measured optical thickness of the artificial cornea. It is clearly visible that the optical thickness increases drastically from $1109 \mu \mathrm{m}$ (1653 $\mu \mathrm{m}$ physical thickness) to $1199 \mu \mathrm{m}$ directly after the application of an unknown quantity of oil. It then follows a negative exponential trend until it levels off at $1114 \mu \mathrm{m}$, $5 \mu \mathrm{m}$ thicker than the measured optical thickness of the clean cornea (first measurement point).

An extract of a temporal series of scans through the artificial cornea before and after the immersion oil has been applied is shown in Figure 4. A typical scan through the clean cornea shows a large reflection from the anterior surface and a slightly smaller reflection from the posterior surface (Figure 4(a)). Directly after the oil has been applied, both reflections vanish completely (b). It is assumed that the oil drop on the cornea distorts the light too much to produce a reflection good enough to subsequently pass through the confocal pinhole. 4(c) shows how the reflection from the posterior surface of the cornea returns - still highly distorted - and in (d) the reflection from the anterior surface reappears, showing a small shoulder peak from the reflection from the oil film and a slightly higher reflection from the anterior surface of the PMMA cornea as well as a slightly distorted reflection from the posterior surface. In (e) most of the oil has flowed off and both reflections are - though still slightly distorted - coming back towards the original higher values. The last plot (f) shows the final state of the system where the residual oil has dried and the amplitudes of the reflections are close to the ones from the original clean cornea. Although no truly repeatable results could be obtained due to the quantity of oil applied being unknown, the results shown here are representative for all repetitions of the experiment.

\section{Typical scan (confocal instrument)}

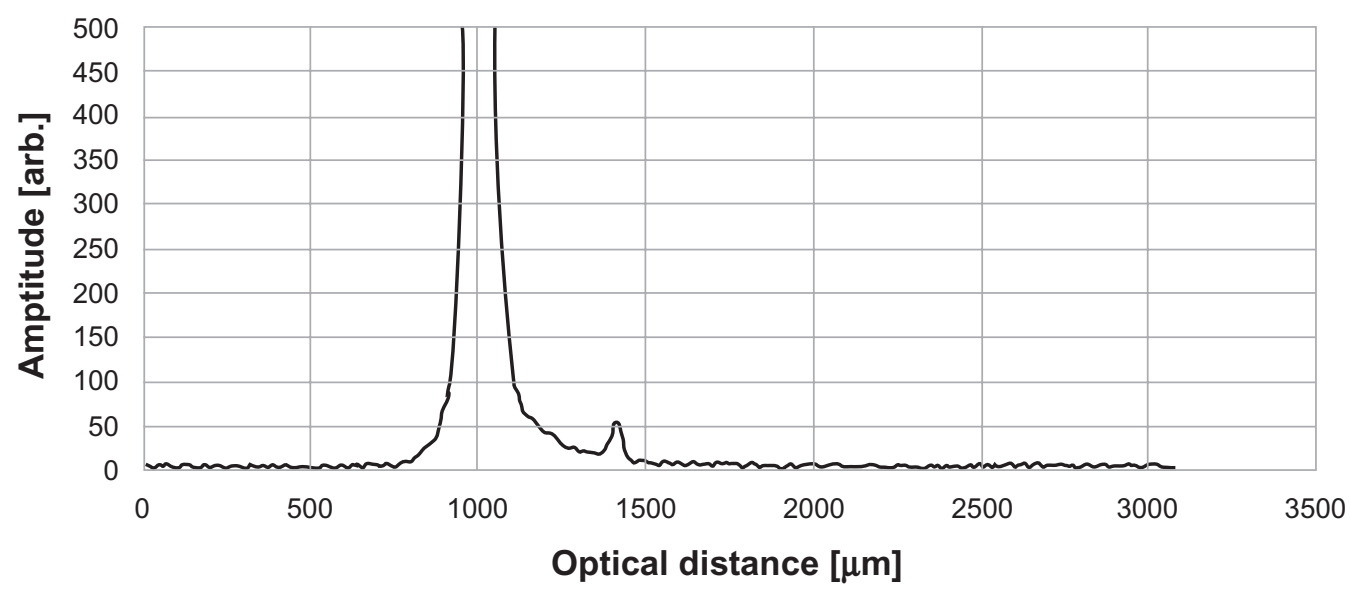

Figure 2 A typical scan of the cornea with the confocal instrument showing a large reflection from the anterior surface and a smaller reflection from the posterior surface. 


\section{Optical thickness of cornea}

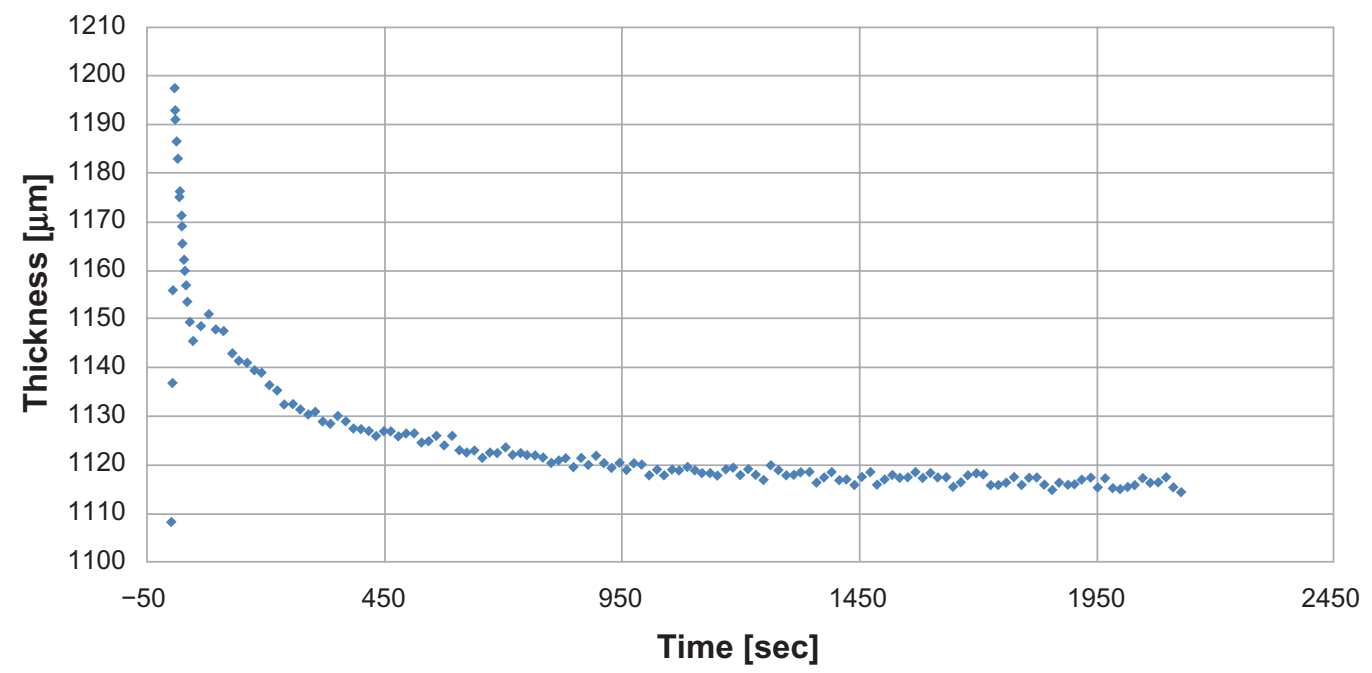

Figure 3 This graph shows the change of the optical thickness of the artificial cornea over time after the immersion oil has been applied. Directly after the oil has been applied the optical thickness increases drastically to then fall back to its original value after about 25 minutes.

This experiment demonstrates the capability of the new instrument to resolve large as well as small changes in thickness very quickly (one scan was taken in approximately 0.2 seconds, significantly faster than the comparative instrument) and repeatably. In fact, the optical standard deviation of measurements of the clean PMMA cornea was found to be $0.73 \mu \mathrm{m}$. Since we were more interested in the ability to measure relative changes in thickness rather than measuring the absolute thickness the absolute accuracy was not assessed in great detail in this study. The very good standard deviation however shows that the measurements taken with the V350 are very repeatable. The accuracy therefore does not play an important role in the data provided in this paper.
The experiment also shows that the instrument in its current configuration, although not able to measure a film as thin as the tear film, is capable of resolving an oil film as optically thin as $64 \mu \mathrm{m}$.

To examine the performance of a comparative instrument using a different measurement principle (Haag-Streit's LenStar) under realistic circumstances, it was applied to measure the central corneal thickness (CCT) in vivo in a volunteer (female, age 24). To make changes in the corneal thickness visible, Optive ${ }^{\mathrm{TM}}$ lubricating eye drops were applied to the subject's right eye while the left eye was used as reference.

As shown in Figure 5, the application of lubricating eye drops (shown in the graph at 15:22, indicated with an

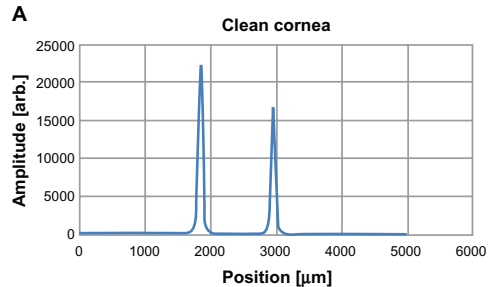

B

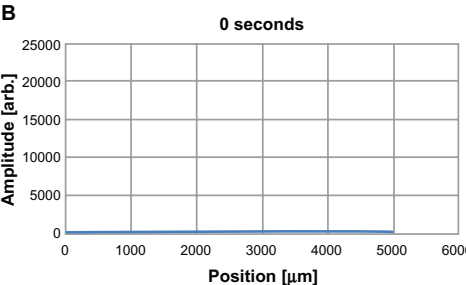

E

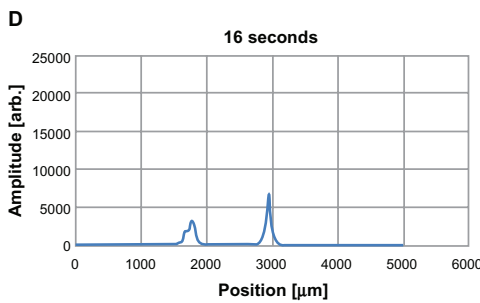

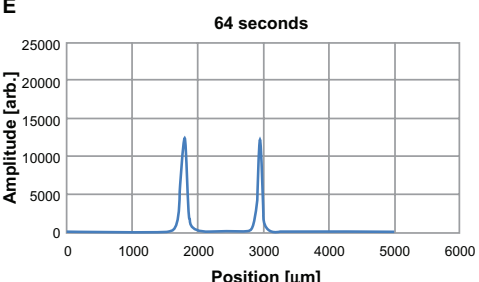

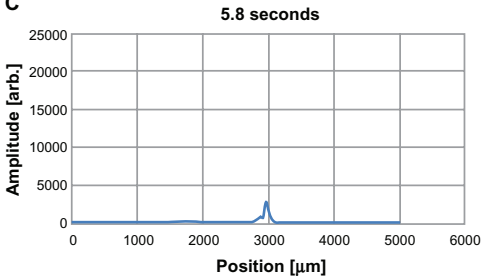

$\mathbf{F}$

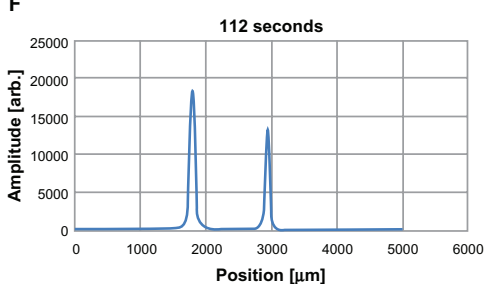

Figure $4 \mathrm{~A}$ temporal series of scans through an artificial cornea showing (a) a typical scan through the clean cornea, (b) the cornea directly after an unknown quantity of immersion oil has been applied, (c) the reflection of the anterior cornea coming back after approximately 6 seconds, (d) and (e) the growing reflection of the anterior cornea (ie, the oil slowly flowing off), and (f) the return to the original state. 


\section{CCT comparison - LenStar}

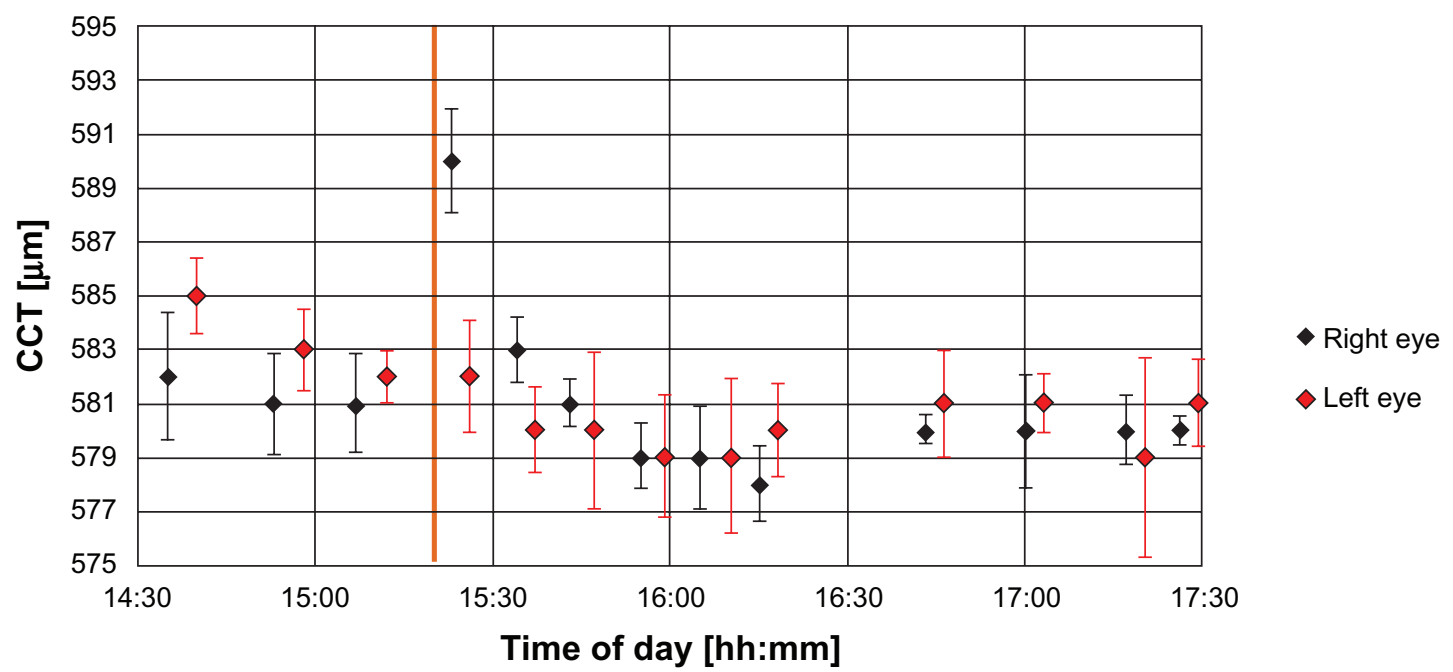

Figure 5 The change in central corneal thickness (CCT) of the subject's right eye after the application of lubricating eye drops measured with the LenStar, also showing the comparison with the left eye of the subject to confirm that the measured increase in corneal thickness is directly related to the eye drops. The orange line at I5:22 indicates the time at which the eye drops were applied.

orange line) leads to an increase of corneal and tear film thickness of $9 \mu \mathrm{m}$. It is also clearly visible that there is no change in thickness in the reference eye. Since the effect of the eye drops wears off rather quickly and the LenStar needs a comparatively long time (up to a few minutes) to acquire one measurement of the corneal thickness no detailed monitoring of the eye drop induced changes in corneal thickness was possible.

The thickness of the cornea in the reference eye drops constantly over the time of the measurement as expected. The LenStar, however, is not capable of resolving the tear film from the cornea. It is, as most other instruments available on the market, configured to measure the corneal and lens thickness and other features in the eye such as astigmatism. Interferometric methods are in general not well suited for measuring the tear film thickness and break up time due to the optical complexity of the eye. Furthermore the data acquisition time required by current interferometric instruments is too long to measure the tear film break up time and more complex data analysis is required to interpret the results.

\section{Discussion and conclusion}

The measurements presented in this paper show the performance of the newly developed confocal instrument in measuring the corneal thickness of an artificial eye. To demonstrate the performance under changing circumstances and simulate the tear film, immersion oil was applied to the artificial cornea. The results show that the new instrument measures the thickness of the artificial cornea with a standard deviation of less than $1 \mu \mathrm{m}$. They also demonstrate that the instrument is very capable of accurately resolving fast, large as well as small changes in corneal thickness and able to clearly resolve films of an optical thickness of $64 \mu \mathrm{m}$. Since this version of the instrument was initially configured to measure the thickness of the cornea and lens, it is not yet capable of resolving the tear film, but the results presented on the oil measurements suggest that when reconfigured the new instrument will perform just as well in measuring the tear film thickness and break up time.

Other methods to resolve optical features in the eye, such as optical coherence tomography, exist. ${ }^{11}$ These methods are, however, much more costly and also use more complicated techniques to acquire the images and data than the confocal instrument. Furthermore, confocal microscopy is known for its very good axial resolution when measuring in nonscattering tissues such as the cornea. ${ }^{12}$ Although the new instrument was not configured to measure the thickness of the tear film it was capable of accurately resolving the thickness of an oil film on an artificial cornea.

It is therefore believed that the newly developed confocal instrument and its later generations which will be configured to accurately resolve the human tear film in vivo will play a crucial role in clinical applications which demand small, accurate, and cost effective solutions. The instrument will be further improved to include the ability to measure fluorescence in the eye and also to monitor the polarization of the returned signal. Thus it is hoped that the instrument will be able to provide information on the internal structure of 
the cornea. Such information will be very useful to monitor changes in the cornea either as a result of refractive surgery or during complications of glaucoma, as well as satisfying the current interest in the tear film to cornea interface.

The next steps in the development of the confocal instrument are to increase the axial resolution of the instrument to enable us to resolve the tear film. Ethical and MHRA regulatory approval will then be sought for a clinical trial which will allow us to evaluate changes in human corneal thickness, also in relation to applanation tonometry, and to measure the tear film thickness and break up time under varying conditions.

\section{Acknowledgment}

Daniel Daly is a shareholder in Lein Applied Diagnostics who are developing the confocal instrument. Kim Buttenschön acknowledges support from the Engineering and Physical Sciences Research Council.

\section{Disclosure}

The authors report no conflicts of interest in this work.

\section{References}

1. Berufsverband der Augenärzte Deutschlands e.V., Deutsche Ophthalmologische Gesellschaft. Das Trockene Auge - eine ernstzunehmende Krankheit. Augenärzte informieren. 2007;Sep:1-11.

2. Oyster CW. The human eye - structure and function. Sunderland, MA: Sinauer Associates, Inc; 1999.
3. Albarrán C, Pons AM, Lorente A, Montés R, Artigas JM. Influence of the tear film on optical quality of the eye. Contact Lens Ant Eye. 1997; 20(4):129-135.

4. Korb DR, Craig J, Doughty MJ, Guillon JP, Tomlinson A, Smith G. The tear film-structure, function and clinical examination. 2002. Rochester, UK: Butterworth-Heinemann; 2002.

5. Tutt R, Bradley A, Begley C, Thibos LN. Optical and visual impact of tear break-up in human eyes. Invest Ophthalmol Vis Sci. 2000;41: 4117-4123.

6. Lemp MA, Foulks GN. The Definition and Classification of Dry Eye Disease. Guidelines from the 2007 International Dry Eye Workshop. Bethseela, MD: National Eye Institute; 2008.

7. Schaumberg DA, Sullivan DA, Buring JE, Dana MR. Prevalence of dry eye syndrome among US women. Am J Ophthalmol. 2003;136(2): 318-326.

8. National Eye Institute. Facts About Dry Eye. National Eye Institute; 2009.

9. National Institute for Health and Clinical Excellence. Glaucoma diagnosis and management of chronic open angle glaucoma and ocular hypertension. London, UK: NICE Clinical Guideline; 2009.

10. Rousseau C, Poland S, Hall AF, Whitters CJ, Girkin JM. Development of fibre-optic confocal microscopy for detection and diagnosis of dental caries. Caries Res. 2007;41:245-251.

11. Wang J, Fonn D, Simpson TL, Jones L. Precorneal and re- and postlens tear film thickness measured indirectly with optical coherence tomography. Invest Ophthalmol Vis Sci. 2003;44(6):2524-2528.

12. Pawley JB, editor. Handbook of Biological Confocal Microscopy. 2nd ed. New York, NY: Plenum Press; 1995.
Clinical Optometry

\section{Publish your work in this journal}

Clinical Optometry is an international, peer-reviewed, open access journal publishing original research, basic science, clinical and epidemiological studies, reviews and evaluations on clinical optometry. All aspects of patient care are addressed within the journal as well as the practice of optometry including economic and business analyses. Basic and clinical

Submit your manuscript here: http://www.dovepress.com/clinical-optometry-journal

\section{Dovepress}

research papers are published that cover all aspects of optics, refraction and its application to the theory and practice of optometry. The manuscript management system is completely online and includes a very quick and fair peer-review system, which is all easy to use. Visit http://www.dovepress. com/testimonials.php to read real quotes from published authors. 